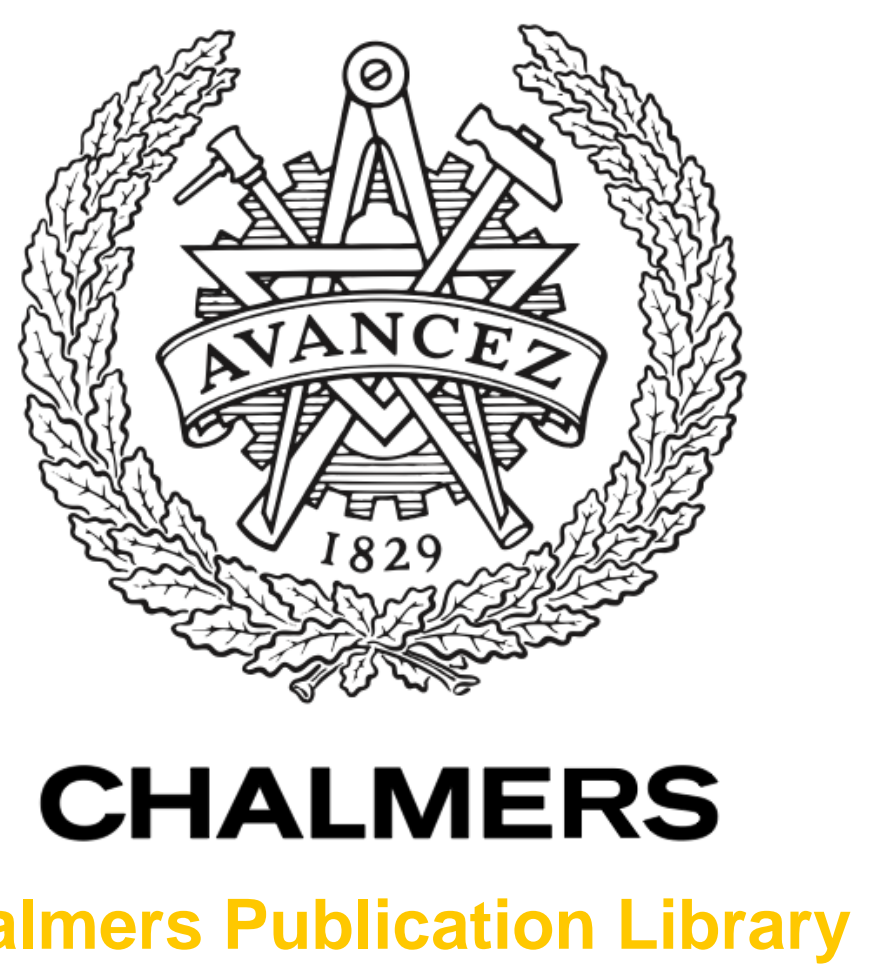

Effect of calcium neutralization on elastic and swelling properties of crosslinked poly(acrylic acid) - correlation to inhomogeneities and phase behaviour

This document has been downloaded from Chalmers Publication Library (CPL). It is the author's version of a work that was accepted for publication in:

E-POLYMERS (ISSN: 1618-7229)

Citation for the published paper:

Larsson, M. ; Gustafsson, S. ; Olsson, E. (2009) "Effect of calcium neutralization on elastic and swelling properties of crosslinked poly(acrylic acid) - correlation to inhomogeneities and phase behaviour". E-POLYMERS, vol. 9(1), pp. 1683 â1696.

Downloaded from: http://publications.lib.chalmers.se/publication/108014

Notice: Changes introduced as a result of publishing processes such as copy-editing and formatting may not be reflected in this document. For a definitive version of this work, please refer to the published source. Please note that access to the published version might require a subscription. 


\title{
Effect of calcium neutralization on elastic and swelling properties of crosslinked poly(acrylic acid) - correlation to inhomogeneities and phase behaviour
}

\author{
Mikael Larsson, ${ }^{1}$ Stefan Gustafsson, ${ }^{2}$ Eva Olsson, ${ }^{2}$ Anette Larsson ${ }^{1 *}$ \\ $1^{*}$ Department of Chemical and Biological Engineering, Chalmers University of \\ Technology, 41296 Göteborg, Sweden; fax: +46 3177234 11; e-mail:
} anette.larsson@chalmers.se, mikael.larsson@chalmers.se

${ }^{2}$ Department of Applied Physics, Chalmers University of Technology, 41296 Göteborg, Sweden; fax: +46 3172232 24; e-mail: eva.olsson@fy.chalmers.se, stefan.gustafsson@chalmers.se

(Received: 14 November, 2008; published: 26 December, 2009)

\begin{abstract}
Crosslinked hydrogels of poly(acrylic acid) neutralized with calcium hydroxide were synthesized using free radical co-polymerization. The effects of the amount added calcium on the elastic modulus and swelling properties of the gels and the correlation to phase behaviour and structural changes within the gels were studied using texture analysis, gravimetric analysis, scanning electron microscopy (SEM), energy dispersive X-ray (EDX) analysis and atomic force microscopy (AFM). It was found that the elastic modulus decreased nonlinearly with increasing amount of added calcium and that the swelling's dependence on the amount of added calcium was complicated. The maximum swelling increased with increasing amount of added calcium until a critical calcium content was reached, upon which the gels phase separated, with a strong decrease in swelling as a result. The changes in properties are explained by the fact that calcium affects the structure of the polymer network during synthesis and by the phase behaviour of the gels. Changes in the gel structure with the addition of calcium were detected with AFM. Furthermore, AFM revealed different phases on the nanometre scale for the sample with a calcium content around which phase separation is macroscopically observed. Finally, it was shown that the sulphur from the initiator potassium persulfate formed crystal like regions with high sulphur and calcium content upon drying of the hydrogels.
\end{abstract}

\section{Introduction}

Hydrogels are materials that lately have been subjected to extensive studies due to their wide range of applications. They can be used in pharmacy as drug delivery systems, in hygiene products as absorbents, in biotech as ion-exchange resins, in engineering as self repairing seals, among other applications [1-3]. Poly(acrylic acid) (PAA) is commonly used to form hydrogels because acrylic acid is cheap and easy to polymerize into high molecular weight compounds and because of its ability to absorb many times its own weight of water $[1,4]$. The performance of hydrogels in different applications is dependent on the visco-elastic properties, the absorbance capacity and the swelling kinetics of the gels. These properties are strongly affected by the degree of crosslinking. It has been shown that for PAA the equilibrium degree of swelling decreases $[5,6]$ and the elastic modulus increases with an increasing degree of crosslinking [7]. In order to achieve a high swelling capacity PAA is commonly neutralized with sodium, which increases the swelling pressure of the gel 
[8]. An increasing degree of sodium neutralization has also been reported to result in a small decrease in elastic modulus [9].

It has previously been reported that the equilibrium degree of swelling for both PAA and sodium neutralized PAA decreases strongly in solvents containing divalent cations compared to in solvents containing monovalent cations [10,11]. Horkay et al. [10] stated that the reduced swelling is not due to $\mathrm{Ca}^{2+}$ ions acting as stable crosslinks between chains, but rather due to a change in the interactions between the solvent and the polymer chains. However, it has been shown that $\mathrm{Ca}^{2+}$ can stabilize PAA hydrogels by preventing the dissolution of the gel by crosslinking the PAA. This increases the activation energy for relaxation of the polymers [12]. Infrared spectroscopy has distinguished several stable forms of $\mathrm{Ca}^{2+}$ binding to the carboxyl groups and computer simulations have found two stable conformations of $\mathrm{Ca}^{2+}$ binding to short chains of PAA, one where $\mathrm{Ca}^{2+}$ is coordinated to one or two carboxylate groups and one where the $\mathrm{Ca}^{2+}$ is coordinated through a water molecule [13]. For long PAA chains it has been reported that the presence of calcium ions causes the PAA chains to form coils, until a critical calcium concentration is reached, upon which precipitation of polymer complexes takes place [14].

The interaction of calcium with acrylic acid containing polymers is of interest to study, both because of the need to understand how divalent ions affect ionic polymer networks, and because of commercially interesting applications including; calcium acrylate as grouting material in concrete [15], waste water treatment using $\mathrm{Ca}(\mathrm{OH})_{2}$ in combination with acrylic acid (AA) containing co-polymers [16] and dental cement containing PAA [17].

Polymer networks synthesized by free radical polymerization are inherently inhomogeneous due to non ideal effects such as different and conversion dependent reactivities of the functional groups $[7,18,19,20]$, resulting in fluctuations in the polymer network structure in space. Furthermore, the phase behaviour of the polymerization mixture may change as the reaction propagates, giving rise to phase separated domains or heterogeneities. The degree of heterogeneity is known to increase with the amount of crosslinker [21, 22]. It has been shown that the swelling of hydrogels is strongly dependent on the network structure [23, 24] and that the elastic modulus of hydrogels decreases with increasing inhomogeniety [21, 25]. The decrease in elastic modulus is explained by the formation of areas of high or low crosslink density during the polymerization. The highly crosslinked areas are described as agglomerated microspheres [25] or as clusters [21]. Oguz et al. [21] proposed that the decrease in the elastic modulus is due to the clusters containing a large number of crosslinking molecules as compared to the surrounding network. If the gel is deformed, the crosslinkers within the clusters will not contribute to the observed crosslink density. The clusters will act as single junction points while the surrounding less dense network is deformed, thus resulting in a decrease in elastic modulus.

Given the interactions of calcium ions with PAA that are described above, it seems likely that the presence of calcium during the synthesis of crosslinked PAA would alter the gel structure of the polymerization product. The aim of this paper was to elucidate the effect of calcium ions on PAA hydrogels by studying how the swelling and elastic properties of PAA hydrogels vary with the degree of calcium neutralization performed prior to the synthesis. Also, it was to be investigated if the changes in properties could be connected to changes in the gel structure. Experimental techniques used in this paper were classical mass uptake for studying the swelling of 
the gels, uniaxial compression for studying the elastic properties and atomic force microscopy (AFM), scanning electron microscopy (SEM) and EDX-analysis to perform structure characterization. The results are discussed taking heterogeneities and inhomogeneities of the gels into account.

\section{Results and discussion}

\section{Synthesis}

To study the effect of calcium neutralization on PAA gels samples with different Ca:AA ratios, ranging from 1:4 to pure PAA, were synthesized. The samples were observed during the synthesis reaction in order to determine if any visual differences could be seen between the samples with different Ca:AA ratios. It was found that at the stage during the synthesis reaction where a macroscopic gel started to form the samples with a Ca:AA ratio of 1:6 and 1:4, shifted from transparent to white, whereas the samples with lower calcium content remained transparent. When dried, the samples assumed a glassy appearance, with a tendency of an increasing degree of yellowness with increasing calcium content. However, upon rehydration the samples regained the appearance they had after the synthesis, white for the samples with Ca:AA ratios of $1: 4$ and $1: 6$ and transparent for the other samples.

Opacity is frequently considered to be the clearest sign of heterogeneity or inhomogeniety in samples [26]. Furthermore, it has previously been shown that phase separation is a phenomenon commonly occurring in crosslinking polymerization $[18,25,26]$ and that increasing crosslinker content is an inducing factor $[21,22]$. In the literature it has been suggested that the solubility for PAA gels in $\mathrm{Ca}^{2+}$ containing solution is reduced [10], and that precipitation of PAA occurs in presence of high $\mathrm{Ca}^{2+}$ concentration [14]. It is therefore likely that the neutralization of AA with calcium during the synthesis may induce changed phase behaviour of the mixture and thus alter the structure of the gel product. In particular, the noticeable change in colour and opacity which was observed at Ca:AA ratios of 1:4 and 1:6, is consistent with the precipitation of calcium-acrylate containing regions.

\section{Texture analysis}

To study the dependence of the elastic modulus on the added amount of calcium, samples were subjected to uniaxial compression tests and the resulting force was recorded as a function of compression. The elastic modulus showed a clear dependence on the amount of added calcium, as shown in Figure 1. The modulus decreased with increasing calcium content in the gels, showing an abrupt decrease when the Ca:AA ratio was increased from 1:8 to 1:6. The low elastic modulus for the samples with Ca:AA ratios of $1: 6$ and $1: 4$, as compared to those with less calcium, is in accordance with them being heterogeneous [21].

If the calcium did act as an ordinary cross-linker an increase in the elastic modulus would have been expected. In fact, PAA with a Ca:AA ratio of 1:4 synthesized as described in the experimental section, but without the crosslinking agent N,N'methylenebisacrylamide displays elastic properties similar to the covalently crosslinked samples with the same Ca:AA ratio. In contrast, PAA without added calcium behaves as a highly viscous and adhesive liquid when the crosslinking agent is omitted (result not shown). Thus, it is concluded that calcium can crosslink the gels, but it also induces changes in the network structure if present during synthesis. Formation of inhomogeneities in crosslinked gels have been shown to reduce the 
elastic modulus by forming dense regions (clusters) [21, 25]. Clusters with high crosslink density will serve as single junction points and the apparent crosslink density will be decreased [25]. Puig et al. [27] have under controlled forms created gel regions of higher crosslink density than the surrounding macroscopic gel, using polyacrylamide gels. They also reported a decrease in the elastic modulus as the prevalence of densely crosslinked regions was increased. We propose that neutralization with calcium prior to the synthesis causes the synthesis reaction to propagate in a way that favours the formation of densely crosslinked regions and that the crosslinking effect of the calcium can be attributed to the formation of phase separated regions in which the polymer chains are locked in place.

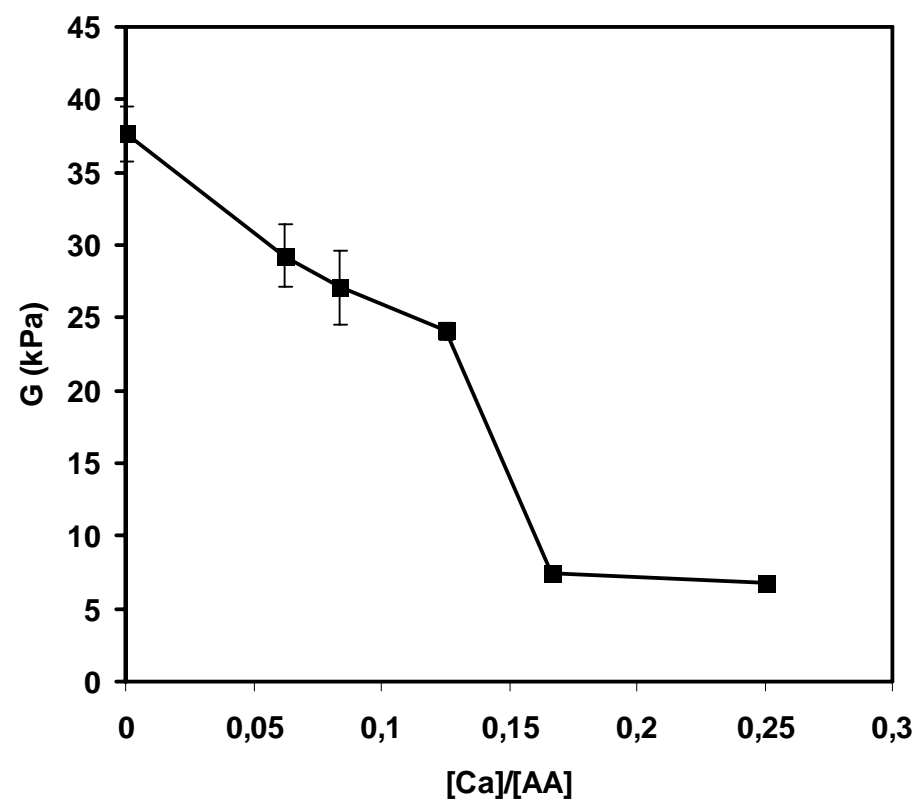

Fig. 1. The elastic modulus as a function of Ca:AA ratio with \pm one standard deviation indicated by error bars $(n=3)$.

\section{Swelling experiments}

In order to study how the swelling properties of the gels varied with the amount of added calcium, the gels were allowed to swell in water and the mass uptake was noted as a function of time. The relation between swelling properties of the hydrogels and the calcium content was found to be complicated. The swelling degree is increased for all times at which the mass uptake was measured for samples with Ca:AA ratios of 1:16-1:8 as compared to pure PAA, whilst for samples with CA:AA ratios of $1: 6$ and $1: 4$ the swelling degree is dramatically decreased as compared to pure PAA (Figure 2).

The equilibrium swelling degree, assumed to be reached at the last measurement time, $t=527$ hours, increased with the amount of added calcium up to a Ca:AA ratio of 1:8, after which it abruptly decreased and showed a converse dependence on the amount of added calcium (Figure 2b). Although it can be seen from Figure 2a that equilibrium is not yet achieved for the sample with a Ca:AA ratio of 1:8, the swelling degree at that time can be regarded as representative to the equilibrium value for the sake of reasoning. 


\section{a}
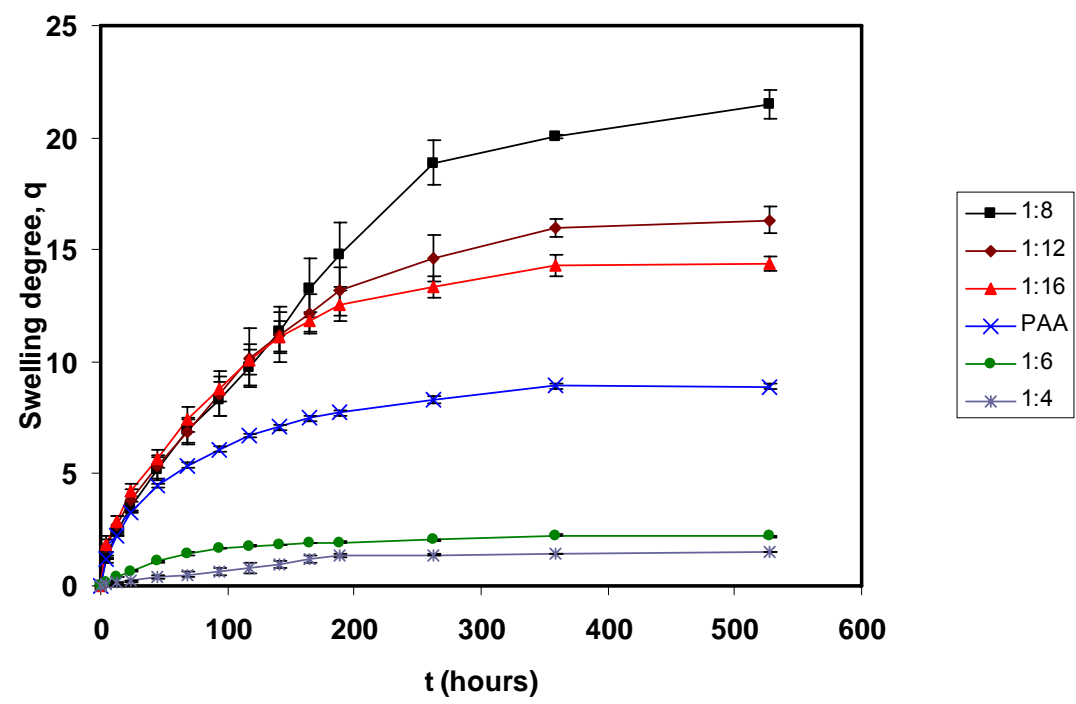

b

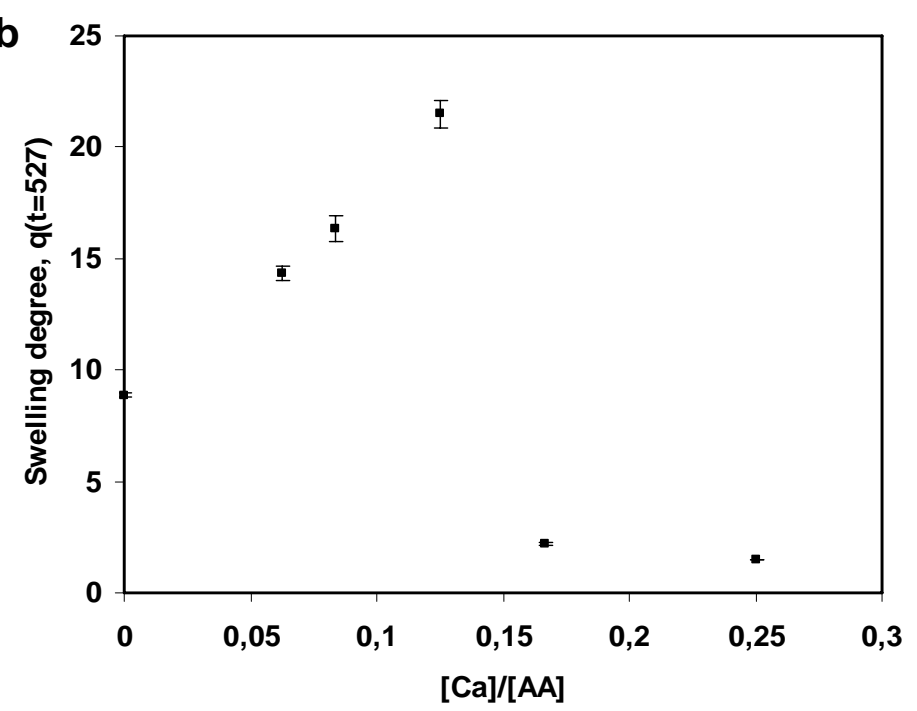

C
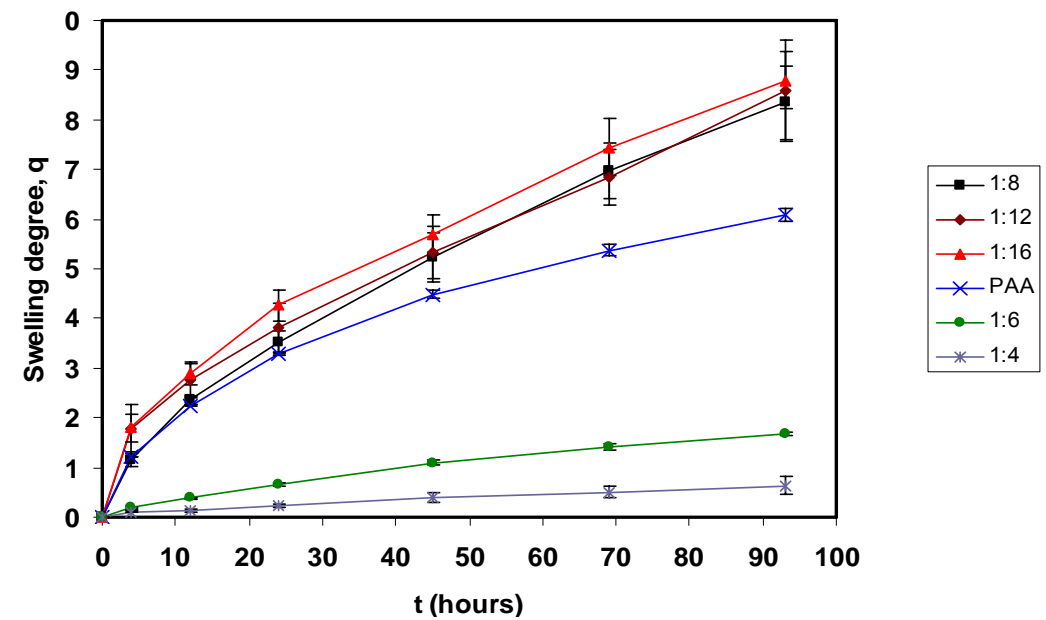

Fig. 2. Swelling degree (q) in Milli-Q water for samples with different Ca:AA ratios. a) as a function of time, b) at $t=527$ hours, taken as equilibrium time. c) for times $\leq 93$ hours, \pm one standard deviation indicated by error bars $(n=3)$. 
In Figure $2 c$ it can be seen that the initial swelling rate for the samples with a Ca:AA ratio of $1: 16$ and 1:12, is higher as compared to the sample of pure PAA but then decreases with increasing calcium content. An interesting observation is the fact that the sample with a Ca:AA ratio of $1: 8$ actually exhibits an increase in the rate of swelling around 150 hours (Figure 2a), tendencies for such a behaviour may also be seen for the sample with a Ca:AA ratio of 1:12 around 80 hours (Figure 2c).

The swelling capacity of polyelectrolyte gel networks is commonly explained in terms of the osmotic pressure of the gel [28]:

$$
\Pi=\Pi_{\text {mix }}+\Pi_{e l}+\Pi_{\text {ion }}
$$

where $\Pi_{\text {mix }}, \Pi_{e l}$, and $\Pi_{\text {ion }}$ are the contributions to the swelling pressure from the mixing of the polymer chains with the solvent, the elongation of the polymer chains and the non-uniform distribution of counter ions between the gel and the solution, respectively.

To fully explain the complicated swelling dependence on the calcium concentration during synthesis one may take into account the potential crosslinking effect of the calcium ions, which would act by further enhancing the negative contribution to the swelling pressure from the term $\Pi_{e l}$ in Eq. 1. Furthermore, one should also consider potential phase behaviour of the gels. Based on our observations we propose that at low degrees of swelling only a limited amount of calcium acrylate units are soluble in the gel system and contributes to the ionic term in Eq. 1, whilst the insoluble calcium actually crosslink the gel. As swelling continues more of the calcium acrylate becomes soluble and releases $\mathrm{Ca}^{2+}$ ions into the polymer network, which increases the swelling pressure and decreases the crosslinking effect of the calcium. Thus, calcium neutralization with a small amount of calcium would mainly influence the ionic term in Eq. 1. For larger amounts of calcium, all of the calcium ions are not soluble initially. Some ions will crosslink the polymer network and actually decrease the swelling pressure. As swelling continues more of the calcium ions go into solution. This increases the swelling pressure, both by increasing the ionic term, and by decreasing the oppressing crosslinking term in Eq. 1. This explains the observed increase in swelling rate at 150 hours for the sample with Ca:AA ratio of 1:8.

Interestingly, the distance between charges on the polymer chains of the samples with Ca:AA ratios of $1: 8$ and $1: 6$ was approximated by:

$l_{\text {charge }}=\frac{l_{c-c}}{2 e}\left(\frac{[A A]}{[C a]}\right)$

where $e$ is the elementary charge, $l_{c-c}$ is the length of a carbon-carbon bond $(1,54$ $\AA),[A A]$ and $[\mathrm{Ca}]$ are the molar synthesis concentrations of acrylic acid and calcium, respectively. The approximated charge density leads to values of $1 e / 6.16 \AA$ and $1 e / 4.62 \AA$ respectively. The Bjerrum length in water at room temperature is $7 \AA$. Since the approximated distance between charges is less than the Bjerrum length for the sample with $\mathrm{Ca}$ :AA ratio of $1: 8$, it seems likely that around that calcium concentration the effect of non ionizable calcium acrylate groups will begin to contribute to the crosslinking of the gel. Further addition of calcium ions should not increase the ionization degree of the polymer network [29], thus they do not contribute to the term $\Pi_{\text {ion }}$ in Eq. 1. Instead the excess calcium ions will crosslink the calcium acrylates, causing the term $\Pi_{e l}$ in Eq. 1 to increase, effectively reducing the 
swelling of the gels. This explains why the samples with Ca:AA ratios of 1:6 and 1:4 display a low degree of swelling. The non ionizable calcium ions crosslink the polymer network and prevent swelling, regardless of the absorbed amount of water.

\section{Surface Characterisation}

In order to evaluate the influence of calcium neutralization on the structural properties of the polymerization product on the micro and nano scale, the dried gels were characterised by SEM and AFM, respectively.

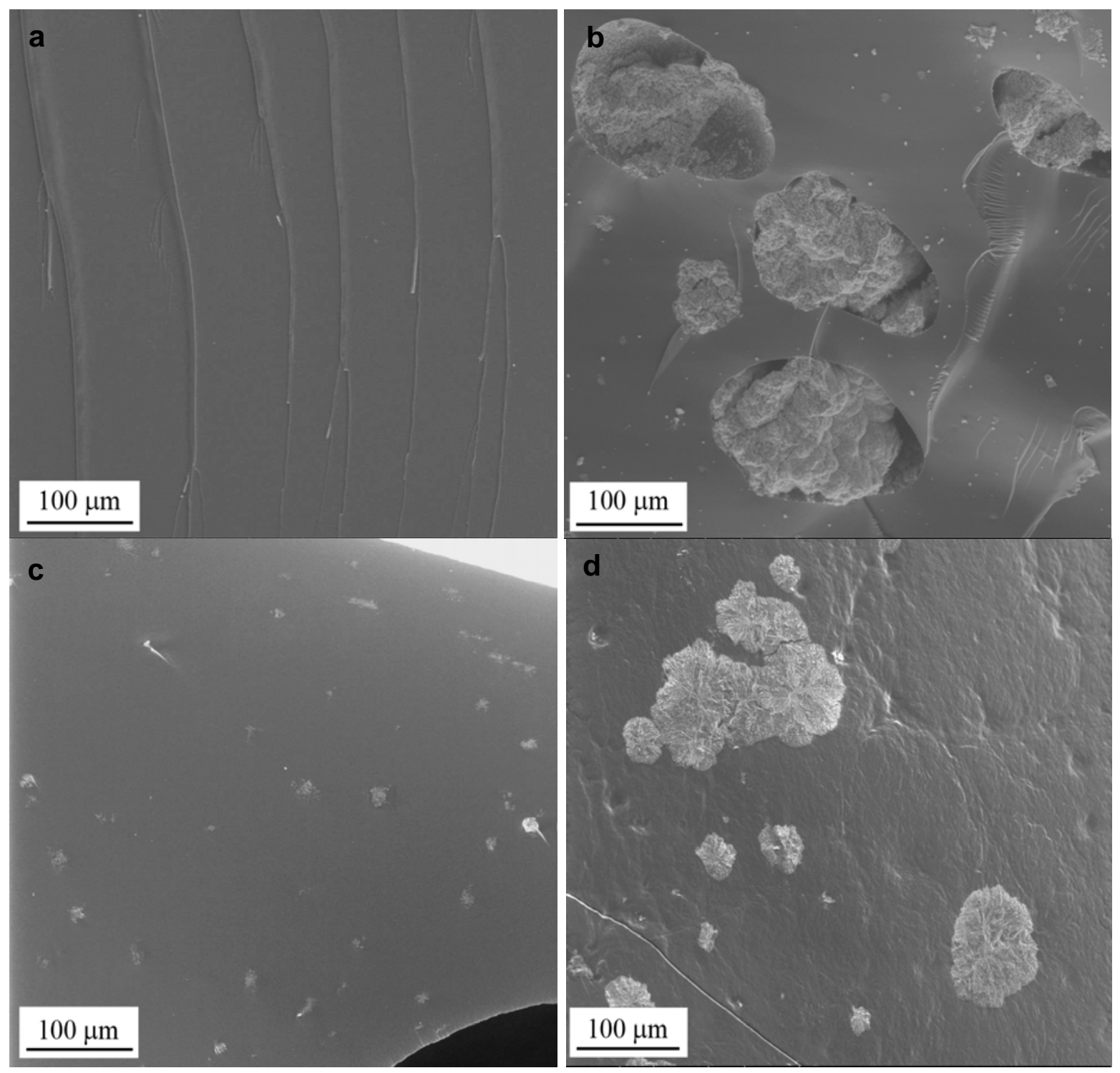

Fig. 3. SEM secondary electron images of the surfaces of dried poly(acrylic acid) with different Ca:AA ratios. a) Pure PAA. b) $C a: A A=1: 8$. c) Ca:AA = 1:4, fractured surface. d) $C a: A A=1: 4$, pore surface.

The SEM images showed that the fractured surface of the pure PAA sample appeared homogenous on the micrometer scale, whereas the samples with calcium contained crystal like regions, ranging in size from approximately a few micrometers up to around two hundred micrometers. In the sample with a Ca:AA ratio of 1:8 those regions were integrated in the gel structure. However, the sample with Ca:AA ratio of 
1:4 had formed a more porous structure upon drying, with the large crystal like regions being present on the pore surfaces (Figure 3).

The fractured surfaces surrounding the crystal like regions appeared to have surfaces with less topographical contrast, similar in appearance to the surface of the pure PAA sample. The patterns, consisting of lines, which are seen on the surface of the pure PAA sample (Figure 3a) are most likely a result of the fracturing of the sample.

Depending on the amount of calcium, the structure of the crystal like regions differed between the samples, as seen at a higher magnification in Figure 4. Many of these regions in the sample with a Ca:AA ratio of 1:4 displayed internal patterns and structures whereas the corresponding regions in the sample with a Ca:AA ratio of 1:8 have a more irregular appearance without any clear internal structure.
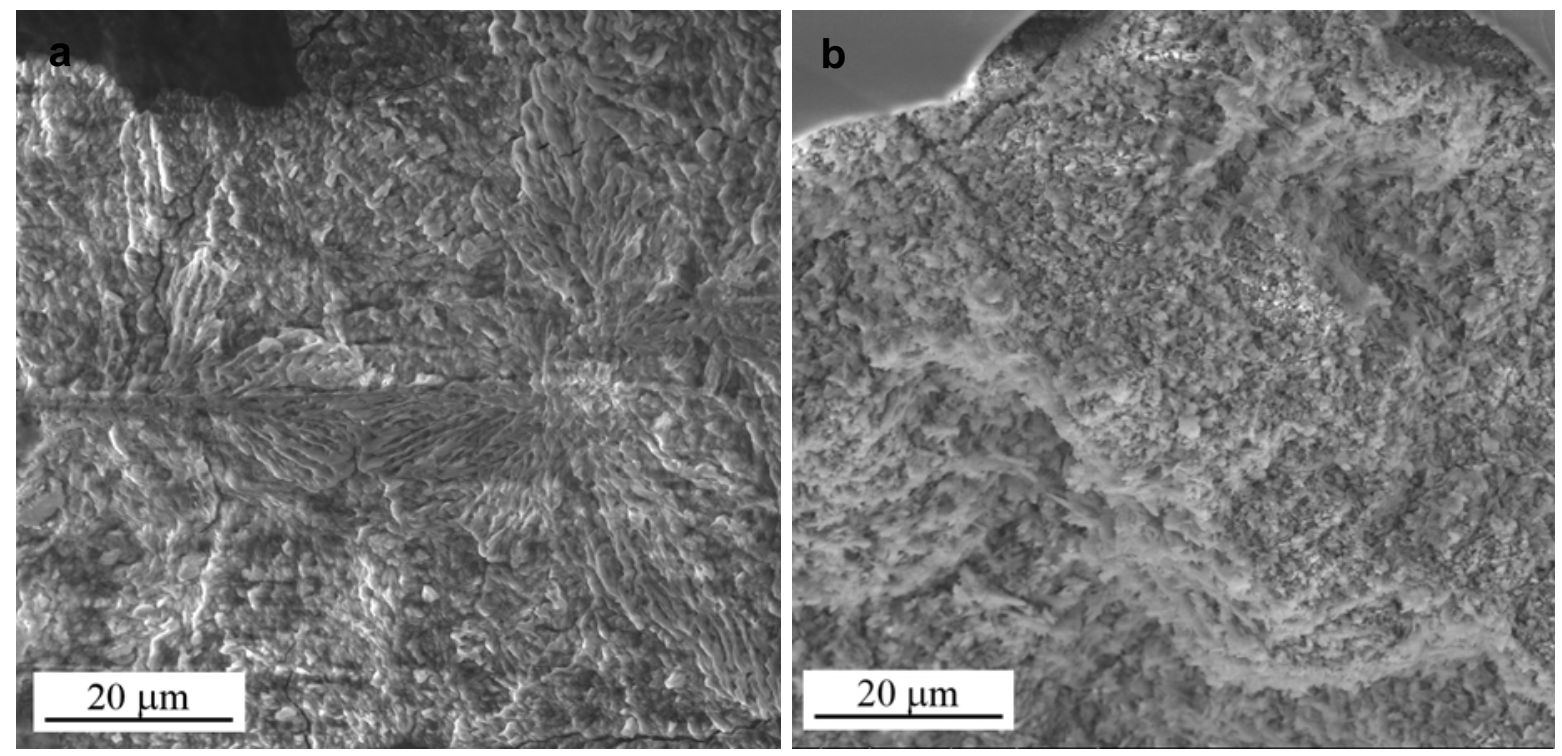

Fig. 4. SEM secondary electron images of the crystal like regions in the samples with a Ca:AA ratio of a) 1:4. b) 1:8.

The fractured surfaces and the crystal like regions of all three samples were characterized with EDX. The composition of the pure PAA sample appeared to be homogeneous throughout the microstructure, with traces of sulphur and potassium from the initiator, in addition to the carbon and oxygen.

It was found that the crystal like regions in the calcium containing samples contained large amounts of sulphur. However, no sulphur was detected in the surrounding areas. The concentration of calcium within the regions was also found to be somewhat higher than in the surrounding volumes. The results from the EDX analysis thus indicate that the sulphur from the initiator potassium persulfate forms regions with high calcium and sulphur contents upon drying, leading to the formation of areas where the polymer network is less dense. The presence of these regions should not affect the swelling or mechanical properties significantly if comparing between the calcium containing samples, this since even for the sample with the lowest Ca:AA ratio of 1:16, the calcium is in excess of the sulphur with more than a $5: 1$ ratio. However, the formation of the complexes could affect the mechanical properties of the calcium acrylate gels since the crystal like regions disrupts the polymer network. For applications where the mechanical properties or the exact structure of the 
network of the dried calcium acrylate are important it may be beneficial to use a different polymerization method or, if practically possible, to wash the product prior to drying.

AFM images of the surfaces acquired after fracturing the samples showed that the surface structure is clearly changed upon the addition of calcium. The sample with pure PAA displayed a surface of elevated spherical structures with a size of about 60 $\mathrm{nm}$. The structures seemed somewhat agglomerated as seen from the phase image in Figure 5a. The samples with Ca:AA ratios up to and including 1:8 also displayed spherical structures. However, the structures were smaller and seemed less agglomerated. In these samples, regions with distinguishably larger and more agglomerated structures were occasionally found (Figure $5 b$ ).

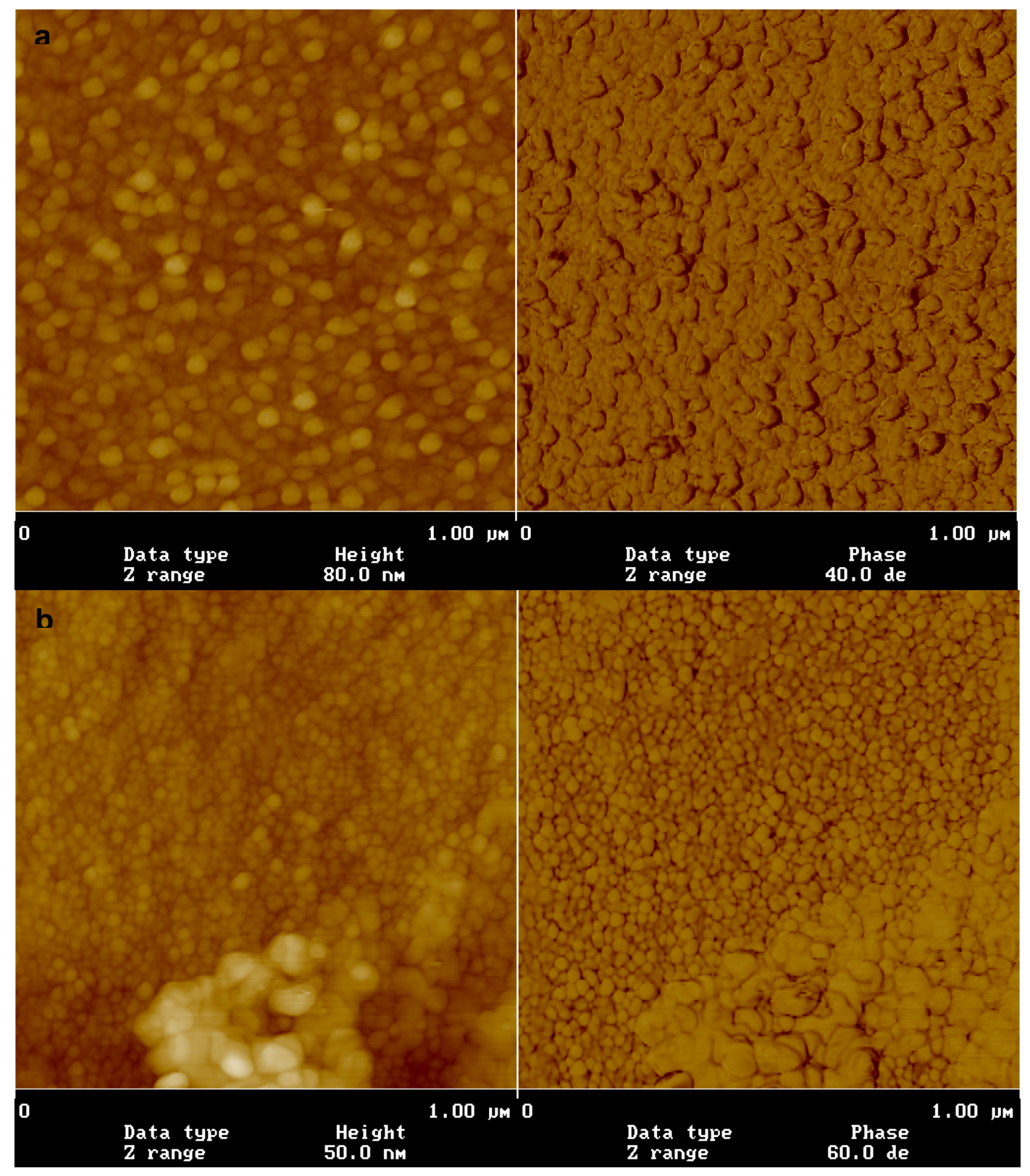

Fig. 5. AFM images of the fractured surface of a) pure poly(acrylic acid) and b) PAA with a Ca:AA ratio of 1:8, with the displacement in the z-direction to the left and the phase image to the right. 
For the sample with a Ca:AA ratio of 1:6, areas corresponding to different phases could be clearly identified, as seen in Figure 6a. In other areas of the sample, regions with a structure similar to that of the samples with less calcium were observed. This is as expected since the areas where phase separation occurred during synthesis should be spread throughout the sample. The possibility that the observed phase differences were an effect of the sulphur containing regions observed with SEM was ruled out, as those areas and their close surroundings were studied specifically without finding any phase differences. Finally the sample with a Ca:AA ratio of 1:4 displayed a surface structure different from all samples with less calcium. No spherical structures were observed, no clear phases were observed and the elevations of the surface displayed an irregular pattern (Figure. 6b).

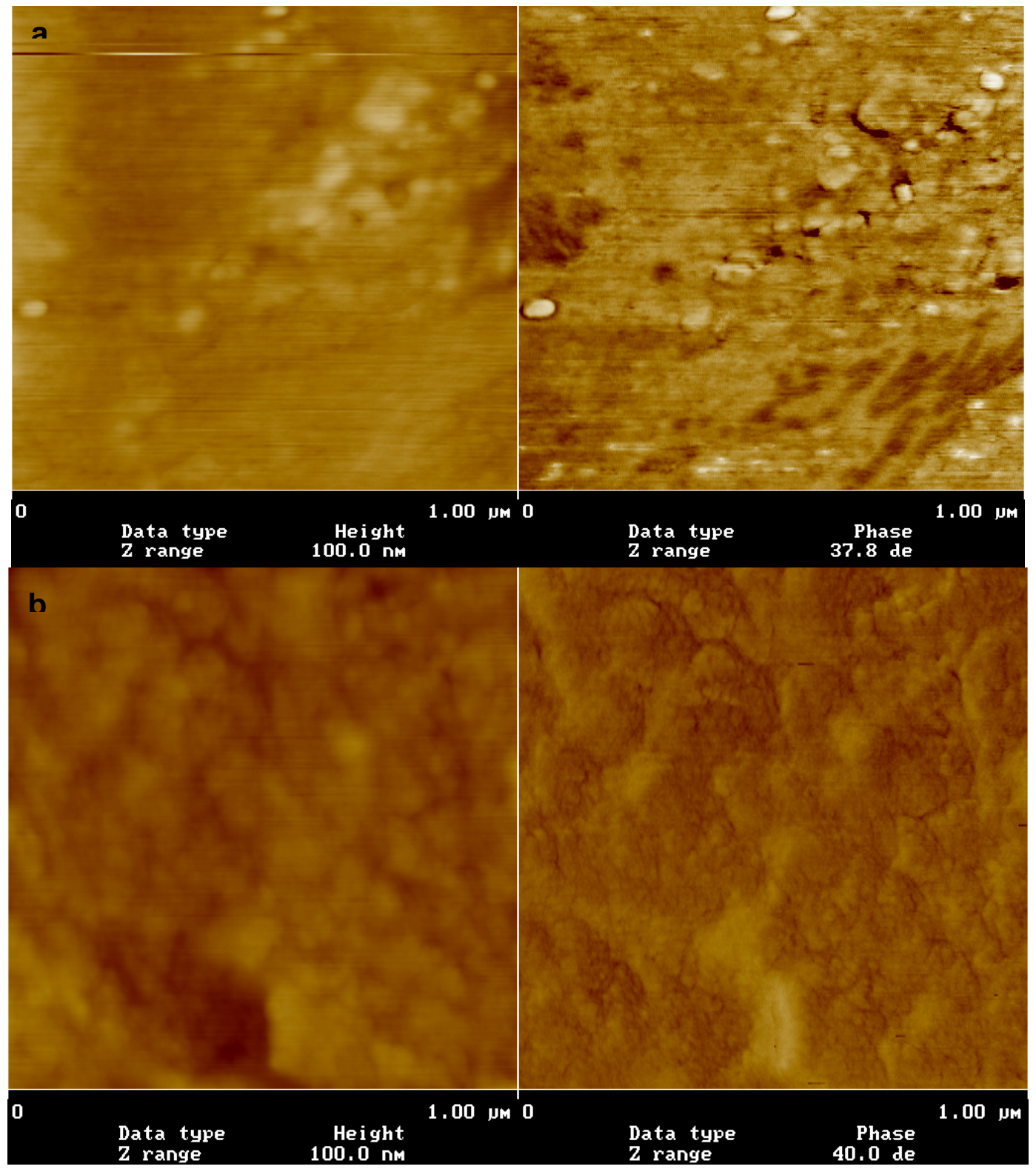

Fig. 6. AFM images of the fracture surface of poly(acrylic acid) with two different Ca:AA ratios, a) 1:6 and b) 1:4, with the displacement in the z-direction to the left and the phase image to the right. 
It may be concluded that the addition of calcium affects the surface properties of the gels in the dry state, and has thus also affected the molecular structure. It seems plausible that the spherical elevations are derived from microgelation during synthesis, which has been reported for other gel systems [7, 21]. In the literature it has been described that intramolecular crosslinking or cyclization during the initial stages of polymerization may result in compact particles or microgel formation [19]. The decrease in size and the less agglomerated nature of the microgels in the presence of calcium could be due to calcium affecting the formation of those microgels. It seems likely that calcium ions due to their divalent nature enhance nucleation of microgels as well as causing them to become more compact. As the calcium concentration increases, macroscopic phase separation is induced during the gelation. For the sample with a Ca:AA ratio of 1:6 it seems as if the kinetics of the phase separation and polymer network formation are such that different areas of the gel correspond to different coexisting phases with different composition (Figure. 7), whilst for the sample with a Ca:AA ratio of 1:4 the gel seems to be one coherent phase, at least on the length scale of this study. Obviously, since the AFM only scans a small area at each measurement it cannot be ruled out that there are areas with different coexisting phases in the samples with less calcium. However, no such areas were detected.

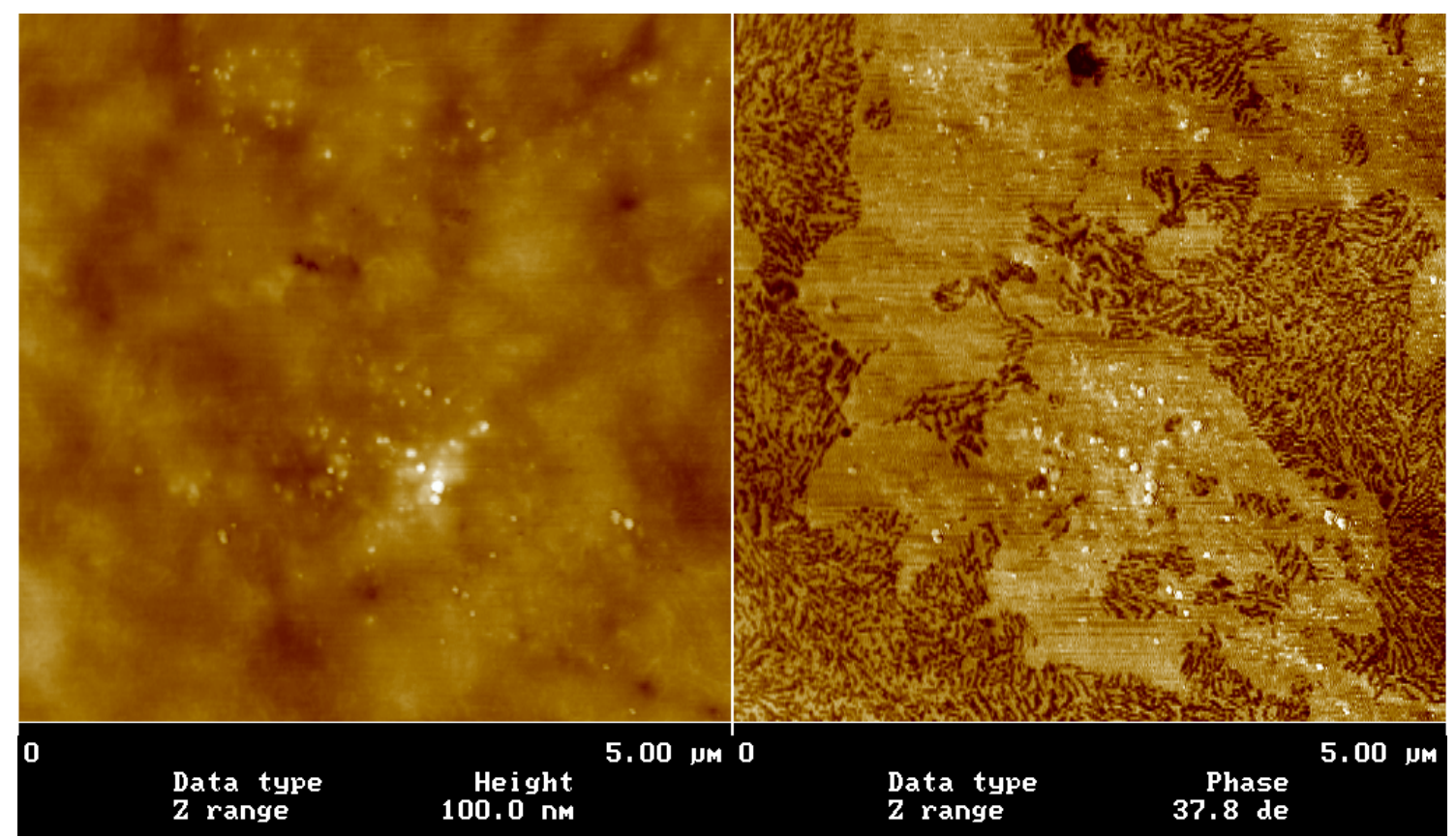

Fig. 7. AFM image of the fracture surface of poly(acrylic acid) with Ca:AA ratio of 1:6, showing the displacement in the z-direction to the left and the phase image to the right.

The observations and reasoning around the results of the AFM study are coherent with those from swelling experiments and texture analysis. It needs to be pointed out, that despite the fact that the dry sample with a Ca:AA ratio of 1:4 appears to be the most homogenous on the studied length scale in AFM, it still is highly heterogeneous when in the gelled state. The sample has undergone a seemingly total phase separation during the polymerization. 


\section{Conclusions}

It has been shown that the neutralization of poly(acrylic acid) with calcium hydroxide prior to synthesis causes significant changes in the swelling and elastic properties of the gels, depending on the Ca:AA ratio of the gels. The changes in both swelling and elastic modulus can be explained by the formation of inhomogeneities and heterogeneities within the gels appeared on a scale of a few micrometer to nanometres, which were confirmed with SEM and AFM. The dramatic decrease in swelling and elastic modulus, as well as a total change of the polymer network structure for high calcium content, is coherent with the fact that the distance between charges becomes smaller than the Bjerrum length. This causes any additional calcium to crosslink the PAA polymers, leading to phase separated gels. Finally, it was shown that the potassium persulfate initiator causes formation of crystal like areas with high concentration of calcium and sulphur. To the authors' knowledge, the above results have not been previously reported and hopefully they can prove valuable in future applications where the interaction of PAA with divalent ions is of importance. Future applications could be synthesis in the presence of divalent ions to create network structures not otherwise achievable or to create ionically crosslinked gels. The divalent ions could then be removed through ion-exchange, leading to gels with different properties or to a swelling and erosion behaviour which is dependent on the surrounding ionic strength. Additionally, interactions of polyvalent molecules with charged hydrogel networks are highly relevant in understanding the release of drug molecules from such delivery systems, something that currently is subject to much research.

\section{Experimental part}

\section{Materials}

All of the following chemicals were of analytical grade and were used as received; Acrylic acid (Fluka, Belgium), calcium hydroxide (Riedel de Haen, Germany), potassium persulfate (Sigma-Aldrich, Germany), N,N,N',N',-tetramethylenediamine (Fluka, Switzerland), N,N'-methylenebisacrylamide (Sigma-Aldrich, Germany).

\section{Synthesis}

Hydrogels containing $25 \mathrm{w} / \mathrm{v} \%$ PAA with a crosslink degree of $0.5 \%$ were synthesized by free radical copolymerization as follows: $3.97 \mathrm{~g}$ Milli-Q water, $3.00 \mathrm{~g}$ acrylic acid, $1.60 \mathrm{~g} 2 \mathrm{w} / \mathrm{v} \% \mathrm{~N}, \mathrm{~N}$ '-methylenebisacrylamide and $3.38 \mathrm{~g} 2 \mathrm{w} / \mathrm{v} \%$ potassium persulfate were mixed on ice. The mixture was neutralized with calcium hydroxide to molar ratios $\mathrm{Ca}: \mathrm{AA}=(0,1: 16,1: 12,1: 8,1: 6,1: 4)$. After neutralization the samples were stirred on ice for 30 minutes, followed by sonification in iced water for an additional 30 minutes. To each sample $50 \mu \mathrm{l}, \mathrm{N}, \mathrm{N}^{\prime}, \mathrm{N}^{\prime}$,-tetramethylenediamine was added under gentle stirring to initiate the polymerization reaction. The samples were then immediately transferred to vials with a diameter of $12 \mathrm{~mm}$, which were placed in a water bath at $60{ }^{\circ} \mathrm{C}$ for $2 \mathrm{~h}$. Finally the samples were allowed to settle for $24 \mathrm{~h}$ at room temperature before analysis.

\section{Texture Analysis}

The gels were cut into cylinders with a height of $15 \mathrm{~mm}$. Uniaxial compression tests were performed in order to determine the elastic modulus of the different samples. The samples were compressed to about $50 \%$ of their initial length at a rate of 0.1 
$\mathrm{mm} \cdot \mathrm{s}^{-1}$ and the resulting force was recorded using a "TA-HDi $囚$, Stable Microsystems", England, with a load cell weight of $5 \mathrm{~kg}$. The compression probe used was a $25 \mathrm{~mm}$ cylindrical aluminium probe, Stable Microsystems, England. Measurements were performed at $19.5 \pm 0.5{ }^{\circ} \mathrm{C}$. The theories for uniaxial compression of Gaussian chains state that [21, 30, 31]:

$P=G\left(\alpha-\alpha^{-2}\right)$

where $P$ is the pressure, $G$ is the elastic modulus and $\alpha$ is the ratio of deformed length to initial length. For deformation ratios up to approximately $20 \%$ the elastic modulus was determined as the slope of the graph $P$ versus $\left(\alpha-\alpha^{-2}\right)$ similar to previous works $[9,10,21,31]$. The non linear data for low strains was discarded as it is derived from imperfect geometries of the sample ends [21, 25].

\section{Swelling Experiments}

Cylindrical samples with a height of $1 \mathrm{~cm}$ were dried for about $200 \mathrm{~h}$ at $40{ }^{\circ} \mathrm{C}$. The swelling experiments were conducted in $650 \mathrm{ml}$ Milli-Q water $(18.2 \mathrm{M} \Omega$ ) at room temperature. The weight of the samples at different times was acquired by placing them on a steel net and touching them briefly with "Kimcare* Medical Wipes, Kimberly-Clark ${ }^{\circledR \Uparrow}$ on the top and bottom to remove excess water before weight measurements. After acquiring the weights the samples were re-submerged. The swelling degree was calculated as:

$q=\frac{\left(w-w_{0}\right)}{w_{0}}$

where $w$ is the recorded weight and $w_{0}$ is the weight of the dry sample.

\section{Scanning Electron Microscopy (SEM)}

Dried gel samples were characterised using a FEI Quanta 200 environmental scanning electron microscope (ESEM) equipped with a field emission gun and an Oxford Inca energy dispersive X-ray (EDX) system for chemical analysis. The samples were fractured prior to characterisation in order to expose their interior structure. The ESEM was operated at a pressure of 0.5 torr in the low vacuum mode in order to avoid charging effects during imaging. An acceleration voltage of $10 \mathrm{kV}$ was used for both imaging and EDX-analysis.

\section{Atomic Force Microscopy (AFM)}

Dried gel samples were characterised using a Digital Instrument Nanoscope Illa with a type G scanner (Digital Instrument Inc. Santa Barbara, CA, USA). The cantilever used was a Micro Masch silicon cantilever NSC 15. The samples were fractured prior to characterisation in order to expose their interior structure. The AFM was operated at a resonance frequency of about $330 \mathrm{kHz}$ in tapping mode, the scan rate was $1 \mathrm{~Hz}$ and the measurements were performed in air.

\section{Acknowledgements}

We want to thank Anders Mårtensson at Polymer technology, Chalmers University of Technology for his help with the AFM analysis. This project is part of the VINN Excellence Centre SuMo Biomaterials (Supermolecular Biomaterials - Structure dynamics and properties). The financial support from the Centre is gratefully 
acknowledged. Further financial support was acquired from the Swedish Research Council and from Chalmers Bioscience Program, Chalmers University of Technology.

\section{References}

[1] Liu, Z. S.;Rempel, G.L. J. Appl. Polym. Sci. 1996, 64, 1345.

[2] Jabbari, E.; Nozari, S. Eur. Polym. J. 2000, 36, 2685.

[3] Wack, H.; Ulbricht, M. Ind. Eng. Chem. Res. 2007, 46, 359.

[4] Elliott, J.E.; Macdonald, M.; Nie, J. Bowman, C.N.; Polymer 2004, 45, 1503.

[5] Jovanovic, J.; Adnadjevic, B. Polym. Bull. 2007, 58, 243.

[6] Mincheng, Y.; Hongyan, S.; 'Chengshen, Z.; Suqin, H. Nucl. Sci. and Tech. 2007, 18, 82.

[7] Yazici, I.; Okay, O. Polymer. 2005, 46, 2595.

[8] Tong, Z.; Liu, X. Eur. Polym. J. 1993, 29, 705.

[9] Schosseler, F.; Ilmain, F.; Candau, S.J. Macromolecules 1991, 24, 225.

[10] Horkay, F.; Tasaki, I.; Basser, P.J. Biomacromolecules 2000, 1, 84.

[11] Muta, H.; Kawauchi, S.; Satoh, M. Colloid Polym. Sci. 2003, 282, 149.

[12] Joachimiak, A.; Okrasa, L.; Halasmus, T.; Wojciechowski, P. Macromol. Symp. 2005, 222, 203.

[13] Bulo, R. E.; Donadio, D.; Laio, A.; Molnar, F.; Rieger, J.; Parrinello, M. Macromolecules 2006, 40, 3437.

[14] Schweins, R.; Lindner, P.; Huber, K. Eur. Phys. J. 2001, 5, 117.

[15] Han, T. C. J. Zhejiang Univ. 2004, 5, 928.

[16] Jiraprasertkul, W.; Nuisin, R.; Jinsart, W.; Kiatkamjornwong, S. J. Appl. Polym. Sci. 2006, 102, 2915.

[17] Majekodunmi, A. O.; Deb, S. J. Mater. Sci. 2007, 18, 1883.

[18] Okay, O.; Yilmaz, Y.; Kaya, D.; Keskinel, M.; Peckan, Ö. Polym. Bull. 1999, 43, 425 .

[19] Dusek, K. Angew. Macromol. Chem. 1996, 240, 1.

[20] Tobita, H.; Hamielec, A. E. Polymer 1990, 31, 1546.

[21] Durmaz, S.; Okay, O. Polym. Bull. 2001, 46, 409.

[22] Bansil R.; Gupta M. K. Ferroelectrics 1980, 30, 63

[23] Jovanovic, J.; Adnadjevic, B. Polym. Bull. 2007, 58, 243.

[24] Calvino-Casilda, V.; Lopez-Peinado, A. J.; Vaganova, E.; Yitzchaik, S.; Pacios, I.

E.; Pierola, I. F. J. Phys. Chem. B. 2008, 112, 2809.

[25] Sayil, C.; Okay, O. Polymer 2001, 42, 7639.

[26] Calvino-Casilda, V.; Lopez-Peinado, A. J.; Vaganova, E.; Yitzchaik, S.; Pacios, I. E.; Pierola, I. F. J. Phys. Chem. B. 2008, 112, 2809.

[27] Puig, L. J.; Sánchez-Díaz, J. C.; Villacampa, M.; Mendizábal, E.; Puig, J. E.; Aguiar, A.; Katime, I. J. Colloid Interface Sci. 2001, 245, 278.

[28] Aykara, T. C.; Akcakaya, I. Eur. Polym. J. 2006, 42, 1437.

[29] Schosseler, F.; Ilmain, F.; Candau, S. J. Macromolecules 1991, 24, 225.

[30] Treolar, L. R. G. Oxford: Oxford University Press, 1975.

[31] Ozdogan, A.; Okay, O. Polym. Bull. 2005, 54, 435. 